\title{
Microsystems-based Technologies for Air-Pollutant and Gas Detection
}

\author{
Danick Briand \\ Ecole Polytechnique Fédérale de Lausanne (EPFL), Institute of Microengineering (IMT) \\ Sensors, Actuators and Microsystems Laboratory (SAMLAB), \\ Rue Jaquet Droz 1, P.O. Box 526, CH-2002 Neuchâtel, Switzerland \\ danick.briand@epfl.ch
}

\begin{abstract}
We present a review of Microsystems-based technologies for the miniaturization of gas detection systems with a focus on air quality monitoring. On one hand, compared to sensors technologies, an approach based on micro-analytical instruments can fulfill some of the requirements linked to the detection at the trace level of some pollutants with improved selectivity. On the other hand, the detection system becomes more complex at a higher cost which can result in a limited deployment. Some of the Microsystems components to be highlighted in this communication include micromachined gas preconcentrators, gas chromatographs, and infrared spectrometers. Several performing individual components have been reported in the last decade but several issues still remain at the system integration level to get commercially viable and robust products on the market.
\end{abstract}

Key words: Microsystems, MEMS, air pollutants, gas detection, micro-analytical instruments.

\section{Introduction}

Air pollution monitoring is of high concern, especially in large cities. Either to get a wider distribution of air analyzer systems or enable mobile monitoring, a higher level of miniaturization of gas detection systems is desired. Microsystems and MEMS based technologies have enabled the higher integration of gas analyzers demonstrating high sensitivity and good time resolution. Mobile monitoring can be used to investigate natural environment, control air pollution and for safety and security purpose, with the detection of nerve or explosives gases.

Work has been performed on gas sampling, separation columns, detectors, spectrometers, etc, and on the integration of these different components into systems. The miniaturization of the individual components has been addressed with some successes over the past years but the complete system integration remains the main challenge for the emergence of these Microsystems based analytical instruments. Fig. 1 illustrates the different levels of integration of components to form complete analytical systems. Research and developments have been performed for all these three levels with however only few efforts dedicated to the system level aspects and integration.
Looking at these developments based on Microsystems technologies for gas detection, some are more favorable to air quality monitoring, namely gas chromatographs and infrared gas detectors, while others have been specifically developed for security applications, e.g. Ion Mobility Spectrometers. The detection at the trace level of given gaseous species can be supported by the use of a sampling component such as a gas preconcentrator. The trapped species from the preconcentrator can be released in a detection or separation chamber and a response can be obtained from the detectors. The selectivity can be obtained by a separation of the gaseous species in a chromatographic column or by the specific light absorption signatures of molecules in the near and mid infrared spectrums.

We are going to focus in this communication on these two identified technologies for air quality monitoring: miniaturized gas chromatographs (GCs) and infrared (IR) detection systems. We will first proceed to a brief description of Microsystems based solutions proposed with the status on their development. Finally, we will focus on the presentation of some of our work on micromachined gas preconcentrators and FTIR spectrometers which could be combined for the realization of sensitive and selective micro gas analyzers. 


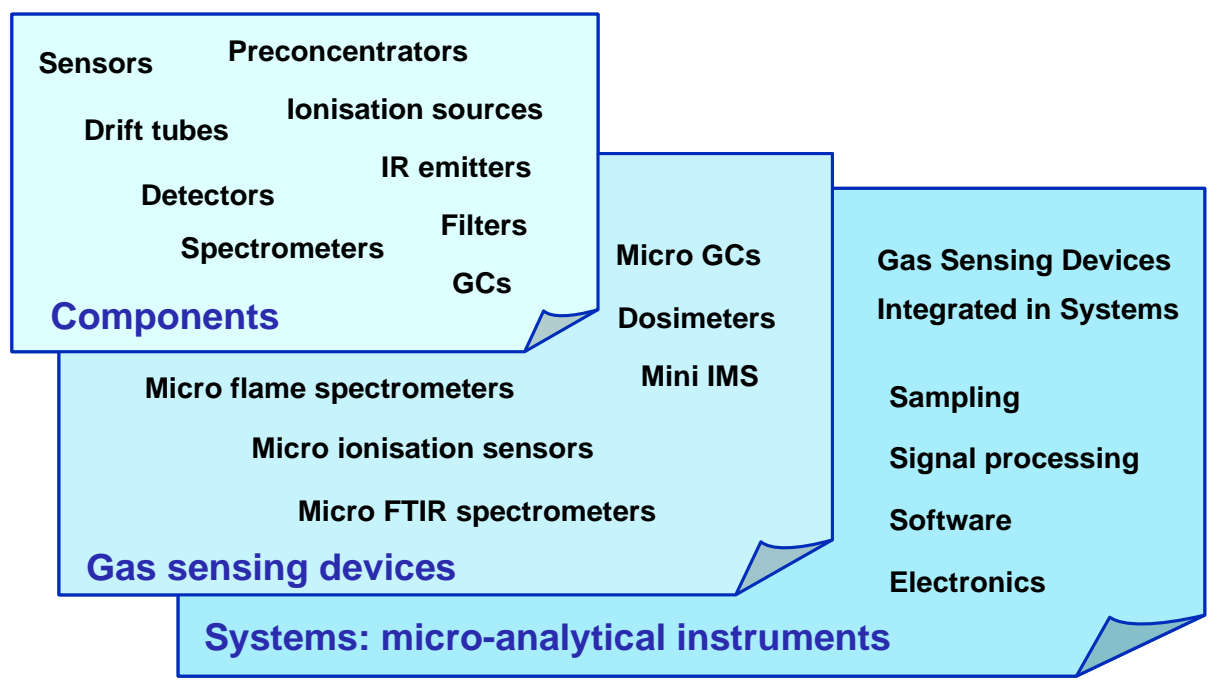

Fig. 1. The different components (not exhaustive list) and levels of integration for miniaturized analytical instruments.

\section{Micro analytical instruments for gas detection}

Efforts have been especially dedicated in USA to the development of micro analytical instruments for gas detection based on gas chromatography. Sandia National laboratory has been a pioneer in the field with their miniaturized Chemlab unit comprising silicon based gas preconcentrators and GCs coupled with surface acoustic wave detectors [1]. Different work has also been produced at the US Naval Research Lab and Virginia Polytechnic Institute and State University on micromachined gas preconcentrators [2,3]. WIMS Center of the University of Michigan has addressed many of the challenges for a complete miniaturized micro-GCs system for gas detection strongly supported by Microsystems technologies [4].

In Europe, different initiatives on the realization of micro-GCs coupled to gas sensors eventually integrating micro gas preconcentrators were launched. Micromachined components and their integration have been reported by CNRIMM Bologna in Italy [5], Université de Franche Comté in France [6], and CSIC-CNM Barcelona - University of Rovira \& Virgili in Spain [7]. In the GOSPEL Network of Excellence on Olfaction and Advanced Gas Sensing Technologies, we have in collaboration with the Ecole des Mines de St-Etienne initiated work on the development of a micro gas preconcentrator [8], which we are now coupled to micro-GCs systems.

Such systems target ppb, even sub-ppb detection levels with analysis time included in between less than 1 minute for explosive detection to 10-15 minutes for environmental monitoring. Power consumption is an issue since heating is required for the gas preconcentrators, the GCs and eventually for the sensors used as detectors, i.e. thermal conductivity detectors (TCD) [9] and metaloxide gas sensors. Pumping and valving components need also to be integrated to the system plus some electronics components. In most cases these components are not Microsystems based, except in the WIMS initiative at the University of Michigan in which pumps and valves were developed, and the main issues remain on their fluidic interfacing with the micro-components and eliminating dead volumes.

Infrared spectroscopy is another interesting method for the detection of gases with high sensitivity, fast response time and good performances in terms of sensitivity. Gas detection is performed by probing the rotationalvibrational transitions of molecules caused by the absorption of IR light. Applications are in process analysis, emissions measurement, environmental monitoring, safety monitoring, and security measurement.

Mid-IR spectroscopy involves IR source, a gas cell, modulating the optical absorption path, and photodetectors. Convential DFB laser diodes operating in the NIR and Quantum cascade lasers for the MIR are sources used. Micromachined hot filament using hotplates technology have been developed has IR source with some limitations in terms of emitted light intensity [10]. There are also non dispersive technologies with filters targeting specific gases. Micromachined thermopiles have also been implemented as detector in a non dispersive configuration [11]. 


\section{Micro gas preconcentrators developed by ARMINES and EPFL-SAMLAB}

The micro gas preconcentrator consists in silicon microchannels filled with an absorbent with capillaries at the inlet and outlet. A screen printed platinum micro-heater on the backside allows heating up the device. In order to have a good regulation of temperature, the micropreconcentrator is suspended in a metallic box. This micro gas preconcentrator is both applicable in the fields of atmospheric pollution monitoring (Volatil organic compounds-VOCs) and explosives detection (nitroaromatic compounds) [12]. Different designs of microdevices and adsorbent materials have been investigated since these two parameters are of importance in the performances of the device. The optimization of the device and its operation were driven by its future application in outdoor environments. Parameters such as the preconcentration factor, cycle time and the influence of the humidity were considered along the optimization process.

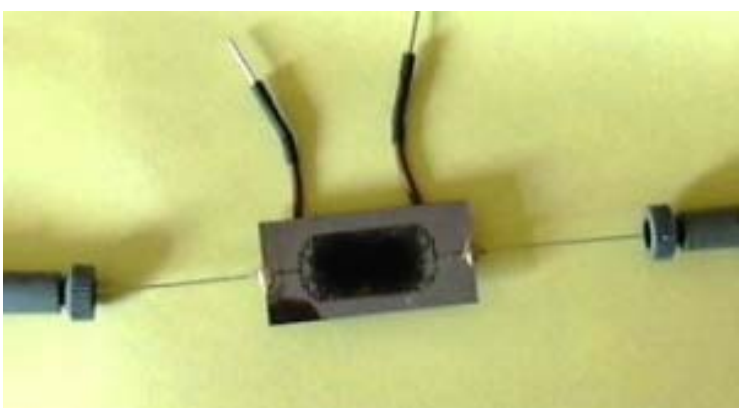

Fig. 2. Image of the silicon micromachined gas preconcentrator filled with carbon nanopowders with fluidic interconnects and electrical connection to the heater screen printed on its backside.

Concerning the absorbent materials, carbon nanopowders, SWCNT and TENAX are used depending on the targeted gases to be preconcentrated. Four main parameters are of importance for the optimum operation of a preconcentrator device with a given geometry and filled with a fixed quantity of specific absorbent material, the gas flow rate during the gas adsorption and the desorption phases, the heating rate during the gas desorption, the duration of the gas adsorption and desorption phases, and the detector (sensitivity and volume of detection). Regarding the optimization of the preconcentration, the adsorption flow was set at $10 \mathrm{NL} / \mathrm{h}$, which allowed us to inject a significant quantity of pollutant in a short time. While the desorption flow was maintained at $2 \mathrm{NL} / \mathrm{h}$ to avoid an important dilution of the desorbed quantity. Using carbon nanopowders and SWCNT, a desorption heating rate of $200^{\circ} \mathrm{C} / \mathrm{min}$ was implemented. Since the desorption is almost complete after 5 minutes, the adsorption and desorption times were maintained constant and last for 5 minutes since for these targeted applications as the pollution monitoring, a short preconcentration cycle is more appropriate.

As a result of this study, a preconcentrator with a total cycle time of $10 \mathrm{~min}$ and the use of single wall carbon nanotubes (SWCNTs) as adsorbent exhibits a good preconcentration factor for VOCs with a limited influence of the humidity.

\section{Micro FTIR gas analyzer developed a EPFL- SAMLAB with ARCoptix.}

We have reported in collaboration with the company ArcOPTIX in Neuchâtel, Switzerland, on the application of a silicon micromachined lamellar grating interferometer in a Fourier transform infrared spectrometer for the detection of gases in the mid infrared region. The spectrometer heart was miniaturized consequently to fit in a box of $30 \times 30 \times 55 \mathrm{~mm}^{3}$ (See Fig. 3). The Fourier transform infrared spectrometer was equipped with mid infrared optical fibers for light coupling.

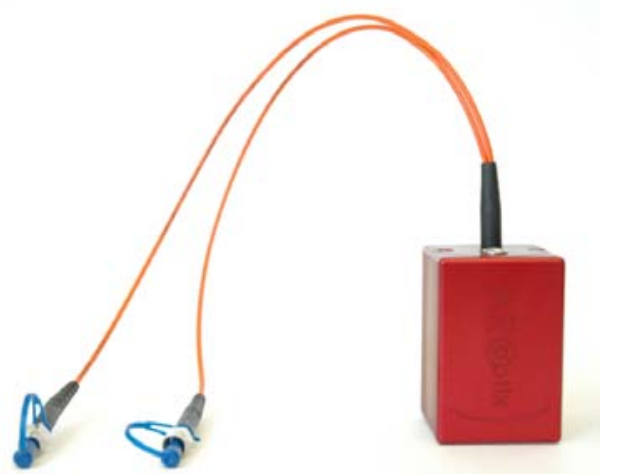

Fig. 3. Top and cross-sectional view of the process flow at foil level and sensor structure.

A conventional gas sensing setup was used comprising a multipass White-cell from International Crystal Laboratories, a halogen lamp, optical fibers and some collimation optics with calcium fluoride lenses. We used a Mercury-Cadmium-Telluride (MCT) detector from VIGO systems with sensitivity between wavelengths of 2 and $5 \mathrm{~m}$. The detector is thermoelectrically cooled down to $-70^{\circ} \mathrm{C}$. Gas measurements in the mid infrared region were focused on specific gases $\left(\mathrm{CO}_{2}, \mathrm{CH}_{4}\right)$ in order to determine the limit of detection and the selectivity that could be obtained using such a micro-spectrometer. Using two different spectral regions we were able to detect concentrations of carbon dioxide over a span from $100 \mathrm{ppm}$ to 9000 ppm with a theoretical detection limit evaluated less than $10 \mathrm{ppm}$. The performed gas measurements showed the proof of principle of a miniaturised Fourier transform infrared gas 
analyzer based on a micromachined spectrometer and fiber optics. More details can be found in Ref. [13].

A multi-wavelengths gas analyzer was set up using FTIR silicon spectrometer with a micromachined lamellar grating. The performance of the system with a long path cell approaches today standards. The micro FTIR suffers form reduced throughput due to consequent miniaturization and uses the concept of massive averaging to get a good signal to noise ratio. A future system needs a larger spectral range and a higher throughput to drive the system in a detector noise limited operation. ARCOPTIX has now come up on the market with a FTIR spectrometer operating in the 2 to $12 \mu \mathrm{m}$ wavelength range with a resolution of $4 \mathrm{~cm}^{-1}$ [14]. Application of signal processing would already now allow to set up a measurement tool for multi-gas analysis.

\section{Conclusion}

MEMS based micro-analytical instruments have surely a strong potential in distributed environmental monitoring applications such as in air pollutants detection. They present obviously some advantages compared to the use of chemical sensors arrays alone and could be deployed as air quality monitoring stations or mobile monitoring solutions. However, applicative systems design and production need to evolve according to the standards in the field of environmental monitoring in a commercially viable way.

\section{Acknowledgements}

We are grateful to the EU FP7 Network of Excellence GOSPEL on Olfaction and Advanced Gas Sensing Technologies for the partial funding of the work presented involving the author.

\section{References}

[1] P.R. Lewis et al., Recent advancements in the gas-phase MicroChemLab IEEE Sensors Journal 6(3) 784-795 (2006); doi: 10.1109/JSEN.2006.874495

[2] I. Voiculescu et al., Micropreconcentrator for enhanced trace detection of explosives and chemical agents IEEE Sensors Journal 6(5), 1094-1104 (2006); doi: 10.1109/JSEN.2006.881431
[3] B. Alfeeli et al., MEMS-based multi-inlet/outlet preconcentrator coated by inkjet printing of polymer adsorbents Sensors and Actuators $B$ 133(1) 24-32 (2008) doi: 10.1016/j.snb.2008.01.063

[4] Q. Zhong, W.H. Steinecker, E.T. Zellers, Characterization of a high-performance portable GC with a chemiresistor array detector, Analyst 134(2) 283-293 (2009) doi: 10.1039/b810944c

[5] S. Zampolli et al., Real-time monitoring of subppb concentrations of aromatic volatiles with a MEMS-enabled miniaturized gas-chromatograph, Sensors and Actuators B 141(1) 322-328 (2009): doi: 10.1016/j.snb.2009.06.021

[6] H. Lahlou et al., Towards a GC-based microsystem for benzene and 1,3 butadiene detection: Preconcentrator characterization, Sensors and Actuators B 156 (2), 680-688 (2011); doi: 10.1016/j.snb.2011.02.018

[7] F. Blanco et al., Fabrication and characterization of microporous activated carbon-based preconcentrators for benzene vapours, Sensors and Actuators B 132(1) 90-98 (2008); doi: 10.1016/j.snb.2008.01.016

[8] E. H.M. Camara et al., Micro gas preconcentrator in porous silicon filled with a carbon absorbent, Sensors and Actuators B 148(2) 610-619 (2010); doi: 10.1016/j.snb.2010.05.054

[9] D. Cruz et al., Microfabricated thermal conductivity detector for the micro-ChemLab ${ }^{\mathrm{TM}}$ Sensors and Actuators B 121(2) 414-422 (2006); doi: 10.1016/j.snb.2006.04.107

[10] J. Hildenbrand et al., Micromachined Mid-infrared emitter for fast transient temperature operation for optical gas sensing systems IEEE Sensors Journal 10(2) 353-362 (2010); doi: 10.1109/JSEN.2009.2034624

[11] R. Rubio et al., Non-selective NDIR array for gas detection Sensors and Actuators B 127(1) 69-72 (2007); doi: 10.1016/j.snb.2007.07.003

[12] E.H.M. Camara et al., A micro gas preconcentrator with improved performance for pollution monitoring and explosives detection, Analytica Chimica Acta 688(2) 175-182 (2011); doi: 10.1016/j.aca.2010.12.039

[13] T. Scharf, D. Briand, S. Buehler, O. Manzardo, H.P. Herzig, N.F. de Rooij, Sensors and Actuators B 147(1) 116-121 (2010); doi: 10.1016/j.snb.2010.03.050

[14] http://www.arcoptix.com/IR_infrared_spectromete r.htm 